\title{
Minor Salivary Gland Inflammatory Lesions in Sjögren Syndrome: Do They Evolve?
}

\author{
Efstathia K. Kapsogeorgou, Maria I. Christodoulou, Demosthenes B. Panagiotakos, \\ Spyros Paikos, Anna Tassidou, Athanasios G. Tzioufas, and Haralampos M. Moutsopoulos
}

\begin{abstract}
Objective. The lymphocytic infiltrates of minor salivary gland (MSG) lesions of Sjögren syndrome (SS) vary in grade and composition and are generally thought to develop in stepwise manner. Their progression over time is not well defined.

Methods. We studied repetitive MSG biopsy specimens from 28 patients with primary SS.

Results. The infiltration grade and prevalence of the major infiltrating cell types ( $\mathrm{T}$ and B cells, macrophages, dendritic cells, natural killer cells) remained largely unchanged during a median 55 month biopsy time interval followup (quartiles 42-81).

Conclusion. We found significant disease progression involving the development of mucosa-associated lymphoid tissue lymphoma in patients expressing adverse serologic prognostic factors, such as low serum C4 complement levels and cryoglobulinemia. (J Rheumatol First Release Aug 1 2013; doi:10.3899/jrheum.130256)
\end{abstract}

Key Indexing Terms:

SJÖGREN SYNDROME MINOR SALIVARY GLAND LESIONS AUTOIMMUNE DISEASES

Sjögren syndrome (SS) is a slowly progressive autoimmune disorder with a broad clinical spectrum that extends from exocrinopathy to diverse systemic manifestations. Evidence of the last 20 years revealed that the glandular and parenchymal extraglandular manifestations are driven by epithelial cells ${ }^{1}$. Glandular dysfunction in SS is associated with the development of periductal inflammatory lesions of variable degree and composition ${ }^{2}$. The composition of the minor salivary gland (MSG) lesions varies according to the infiltrate severity; $\mathrm{T}$ cells predominate in mild infiltrates, B cells in severe infiltrates, whereas the prevalence of

From the Laboratory of Immunology, Department of Pathophysiology, School of Medicine, National University of Athens; Department of Nutrition and Dietetics, Harokopio University of Athens; Dental Department, Laikon General Hospital of Athens; and Department of Hemopathology, Evangelismos Hospital, Athens, Greece.

Supported by grants from the School of Medicine, National University of Athens.

E.K. Kapsogeorgou, PhD, Laboratory of Immunology, Department of Pathophysiology, School of Medicine, National University of Athens; M.I. Christodoulou, PhD, Laboratory of Immunology, Department of Pathophysiology, School of Medicine, National University of Athens; D.B. Panagiotakos, PhD, FRSPH, FACE, Department of Nutrition and Dietetics, Harokopio University; S. Paikos, DDS, Dental Department, Laikon General Hospital; A. Tassidou, MD, Department of Hemopathology, Evangelismos Hospital; A.G. Tzioufas, MD; H.M. Moutsopoulos, MD, FACP, FRCP, MACR, Laboratory of Immunology, Department of Pathophysiology, School of Medicine, National University of Athens.

Dr. Kapsogeorgou and Dr. Christodoulou contributed equally to the study.

Address all correspondence to Dr. H.M. Moutsopoulos, Department of Pathophysiology, School of Medicine, National University of Athens,

75 Mikras Asias street, Athens 11527, Greece.

E-mail:hmoutsop@med.uoa.gr

Accepted for publication May 21, 2013. macrophages increases and interdigitating dendritic cells (iDC) decreases with lesion grade ${ }^{2}$. Although MSG infiltrates are generally thought to develop in a stepwise manner, the progression and change of lymphocytic subpopulations over time have not been studied.

We investigated the progression of autoimmune MSG infiltrates over time, and particularly alterations of the grade and composition of lymphocytic infiltrates, as well as the progression of degenerative processes, such as fibrosis and fatty cell infiltration. Potential associations between histopathological measures of lesion progression and clinical or serological features of patients with SS were examined.

\section{MATERIALS AND METHODS}

Patients and MSG biopsies. Twenty-eight consecutive patients (27 women, 1 man) with primary $\mathrm{SS}^{3}$ who were diagnosed and followed in our department, and had been subjected to their first MSG biopsy at least 36 months previously, were studied. All patients signed a written informed consent. One patient refused to undergo a second biopsy. The study was approved by the Ethics Committee of the School of Medicine, National University of Athens (protocol no. 5107). The median interval between the 2 sequential biopsies was 55 months (quartiles 42-81). In all of the patients with SS, the biopsy focus score of the first MSG biopsy was $\geq 1$. In 24 patients, the first biopsy was performed at diagnosis.

The medical records of patients were evaluated for various clinical, laboratory, and therapeutic variables, as described ${ }^{2}$. None of the patients with SS had evidence of B cell lymphoma, sarcoidosis, hepatitis B virus (HBV), hepatitis C virus (HCV), or HIV infection. Twenty-one patients at the time of the first biopsy had not received immune-modulating agents. None of the 28 patients had received B cell depletion therapy before or during followup. Characteristics of patients with SS and the therapeutic interventions are summarized in Table 1.

Evaluation of progression of autoimmune infiltrates in 2 sequential MSG biopsies. The histological evaluation of repetitive biopsies of at least 4 MSG, with sections stained using hematoxylin/eosin (H\&E), was done

Personal non-commercial use only. The Journal of Rheumatology Copyright @ 2013 . All rights reserved. 
Table 1. Demographic, clinical, and laboratory characteristics of the patients with SS.

\begin{tabular}{|c|c|c|}
\hline Features of Patients with SS & 1st MSG Biopsy & 2nd MSG Biopsy \\
\hline \multicolumn{3}{|l|}{ General } \\
\hline Age, yrs; median (quartiles) & $50.5(36-55)$ & $54.5(42-59)$ \\
\hline Duration of sicca symptoms, yrs; median (quartiles) & $3(1-7)$ & \\
\hline \multicolumn{3}{|l|}{ Clinical } \\
\hline Arthralgias, $\mathrm{n}(\%)$ & $11(39.3)$ & $11(39.3)$ \\
\hline Arthritis, n (\%) & $2(7.1)$ & $3(10.7)$ \\
\hline Salivary gland enlargement, $\mathrm{n}(\%)$ & $12(42.9)$ & $16(57.1)$ \\
\hline Raynaud's phenomenon, n (\%) & $9(32.1)$ & $9(32.1)$ \\
\hline Parenchymal organ involvement, $\mathrm{n}(\%)$ & $0(0.0)$ & $1(3.6)$ \\
\hline Lung involvement, $\mathrm{n}(\%)$ & $0(0.0)$ & $0(0.0)$ \\
\hline Renal involvement, n (\%) & $0(0.0)$ & $1(3.6)$ \\
\hline Liver involvement, $\mathrm{n}(\%)$ & $0(0.0)$ & $0(0.0)$ \\
\hline Indicative of vasculitic involvement, $\mathrm{n}(\%)$ & $4(14.3)$ & $6(21.4)$ \\
\hline Palpable purpura, n $(\%)$ & $4(14.3)$ & $6(21.4)$ \\
\hline Vasculitis, n (\%) & $0(0.0)$ & $1(3.6)$ \\
\hline Peripheral neuropathy, n (\%) & $0(0.0)$ & $0(0.0)$ \\
\hline Lymphoma, n (\%) & $0(0.0)$ & $5(17.9)$ \\
\hline \multicolumn{3}{|l|}{ Laboratory } \\
\hline Leukopenia, n (\%) & $5(17.9)$ & $5(17.9)$ \\
\hline Hyper-gammaglobulinemia, n (\%) & $9(32.1)$ & $9(32.1)$ \\
\hline C4 levels, median (quartiles) & $18.7(12.2-25.1)$ & $21.8(11.4-39.9)$ \\
\hline Anti-Ro(SSA) and/or La(SSB) positive, $\mathrm{n}(\%)$ & $22(78.6)$ & $22(78.6)$ \\
\hline Anti-Ro(SSA) positive, $\mathrm{n}(\%)$ & $22(78.6)$ & $22(78.6)$ \\
\hline Anti-La(SSB) positive, $\mathrm{n}(\%)$ & $12(42.9)$ & $12(42.9)$ \\
\hline Cryoglobulinemia, $\mathrm{n}(\%)$ & $4(14.3)$ & $5(17.9)$ \\
\hline \multicolumn{3}{|l|}{ Therapy } \\
\hline Pilocarpine-hydrochloride, $\mathrm{n}(\%)$ & $0(0.0)$ & $7(25.0)$ \\
\hline Hydroxychloroquine, n (\%) & $2(7.1)$ & $5(17.9)$ \\
\hline Pilocarpine-hydroxychloroquine, n (\%) & $1(3.6)$ & $3(10.7)$ \\
\hline Corticosteroids, n (\%) & $3(10.7)$ & $2(7.1)$ \\
\hline Pilocarpine-corticosteroids, $\mathrm{n}(\%)$ & $0(0.0)$ & $3(10.7)$ \\
\hline Hydroxychloroquine-corticosteroids, n (\%) & $1(3.6)$ & $0(0.0)$ \\
\hline Pilocarpine-corticosteroids-methotrexate, n (\%) & $0(0.0)$ & $1(3.6)$ \\
\hline $\mathrm{B}$ cell depletion & $0(0.0)$ & $0(0.0)$ \\
\hline None, $\mathrm{n}(\%)$ & $21(75.0)$ & $7(25.0)$ \\
\hline
\end{tabular}

blinded by EKK and AGT. To avoid inconsistency due to evaluation of sections at baseline and deeper edges of glands ${ }^{4}$, sections at the middle depth of tissue were routinely evaluated. Further, due to a reported change in the quantification of the focus score in a small percentage of samples with low scoring ${ }^{5}$, sections differing by roughly $200 \mu \mathrm{m}$ were also evaluated in each sample. Similar focus scores were found in the 2 depths evaluated. Progression of the grade of inflammatory MSG lesions was evaluated by measuring the total number of infiltrating mononuclear cells (MNC) $/ \mathrm{mm}^{2}$ tissue, as well as by the standard MSG biopsy scores, according to Chisholm and Mason (biopsy focus score: number of lymphocytic foci $/ 4 \mathrm{~mm}^{2}$ tissue $)^{6}$ and Tarpley, et al $\mathrm{l}^{7}$. Although the Tarpley score is not adequate for MSG biopsy evaluation and diagnosis, it provides information on the infiltrate severity and its association with the destruction of glandular tissue. In a previous report, we classified patients with SS into 3 groups according to lesion severity ${ }^{2}$. In this study, 4 patients with mild, 9 with intermediate, and 15 with severe lesions were included consecutively, suggesting a predisposition for patients with severe MSG inflammation, possibly due to their more frequent followup. None of the standard grading scores (alone or jointly) provides information about the size of the lymphocytic infiltrates, and thus they do not depict changes in the number of infiltrating cells. Therefore, evaluation of the progression of the lesion grade in this study was based primarily on the number of infiltrating $\mathrm{MNC} /$ tissue area. Tissue area and the number of infiltrating MNC in each biopsy specimen were automatically estimated using ImageJ software on serial images of all H\&E-stained sections ${ }^{2}$. Neither of the scores applied was found to be significantly different between the biopsy sections at deeper levels. Degenerative changes, such as fibrosis and fatty-cell infiltration, were also analyzed ${ }^{8}$. The evaluation of germinal center $(\mathrm{GC})$ formation was based on histopathologic criteria and the presence of follicular dendritic cell (fDC) networks ${ }^{2}$.

The change of composition of the MSG infiltrates was assessed by calculating the percentage of each infiltrating cell type [total $\mathrm{T}$ cells and their subpopulations CD4+ T cell, CD8+ T cell, and Treg cells; B cells; macrophages; iDC; fDC; and natural killer (NK) cells] to total infiltrating $\mathrm{MNC}$ in serial sections of the sequential biopsies ${ }^{2}$. MNC types were identified by immunohistochemistry and counted using ImageJ software in all serial sections of paraffin-embedded MSG biopsy specimens, whereas the number of total infiltrating MNC was automatically estimated in each section by the ITCN plug-in (ImageJ software), as described ${ }^{2}$. In preliminary experiments, the percentages of each infiltrating MNC type were found not to differ significantly between sections at deeper levels; however, in all cases, evaluation of the sections at the edges of glands was avoided ${ }^{2}$.

MSG tissues with GC formation or severe infiltration were routinely evaluated for the development of lymphoma by a specialist hemopathologist (AT). The diagnosis and characterization of lymphoma in MSG tissues were assessed by immunohistochemical identification and/or 
molecular detection of B or T cell monoclonality. Immunohistochemical detection was performed with antibodies against specific markers, such as CD20, CD3, CD4, CD8, CD45, CD43, cytoplasmic immunoglobulin (CIg), CD5, CD10, CD15, CD21, CD23, CD35, CD79a, CD30, Bcl-2, Bcl-6, cyclinD1, DBA44, and MIB. Molecular evaluation involved detection of clonal IGH gene rearrangements by PCR using specific primer sets, such as (1) FR3A, LJH, VLGH, and (2) FR2A, LJH, VLGH. Diagnosis of mucosa-associated lymphoid tissue (MALT) lymphoma required the predominance of $\kappa$ to $\lambda$ chains (at least 7 to 1 ratio) and/or detection of monoclonal IGH gene rearrangements.

Statistical analyses. Continuous variables are presented as mean \pm SD (if normally distributed), otherwise as median (quartiles). The significance of the changes observed between the 2 sequential biopsies was analyzed using general linear models for repeated measures (RM ANOVA), adjusted for biopsy time interval. Chi-square test was used to evaluate associations between categorical variables. The limit for considering a change of infiltrating $\mathrm{MNC} / \mathrm{mm}^{2}$ tissue to be significant was arbitrarily set at $60 \%$. Wilcoxon-ranked test was applied to compare patients with SS that presented progression of the MSG infiltrates and those without. Significance was defined as $\mathrm{p}<0.05$. Analyses were done using SPSS version 17.0 and GraphPad-Prism version 5.

\section{RESULTS}

Progression of grade of autoimmune MSG lesions of patients with SS. Data analysis revealed that neither the number of infiltrating MNC/tissue area nor the MSG grading scores (biopsy focus and Tarpley scores) changed significantly during the median biopsy time interval of 55 months' followup (quartiles 42-81; Table 2). Similarly, the other histological variables studied, including GC formation and indicators of tissue degeneration, such as fibrosis and fat infiltration, did not vary significantly in the repetitive MSG biopsy specimens (Table 2). None of the variables was found to be affected by the biopsy time interval or to correlate with demographic, clinical, laboratory, and therapeutic features of patients with SS.

Generally, the severity of the lesion grade remained similar during followup and even in samples with $\geq 60 \%$ change of the infiltrating MNC number/ $\mathrm{mm}^{2}$ tissue between the 2 biopsies; it was not readily evident at microscopic evaluation (data not shown). Six patients presented $\geq 60 \%$ fluctuation of the infiltrating MNC number $/ \mathrm{mm}^{2}$ tissue (increase in 4 patients, decrease in 2; Table 3). In 3 patients, this was accompanied by a change of the biopsy focus and Tarpley scores (Table 3). Except for one patient (Patient 17), these changes were not followed by a change in lesion severity, as assessed by Tarpley score ${ }^{2}$; mild, intermediate, or severe lesions remained the same (Table 3). These alterations were not affected by the biopsy time interval or any of the other histological, demographic, clinical, or therapeutic variables studied. Neo-organization of GC was observed in 4 patients (Patients 1, 13, 15, and 18), but it was not statistically significant. It was not associated with infiltration increase, biopsy time interval, or other histological, demographic, clinical or therapeutic variables (data not

Table 2. Histological features of the successive minor salivary gland (MSG) biopsy specimens of the SS patients. Statistical significance was evaluated by general linear model for repeated measures adjusted for the biopsy time interval.

\begin{tabular}{|c|c|c|c|}
\hline Histological Features of SS Patients & 1st MSG Biopsy & 2nd MSG Biopsy & $\mathrm{p}$ \\
\hline No. infiltrating $\mathrm{MNC} / \mathrm{mm}^{2}$ of tissue, mean $\pm \mathrm{SD}$ & $2774 \pm 2081$ & $3035 \pm 2403$ & 0.67 \\
\hline Glandular area $\left(\mathrm{mm}^{2}\right)$ examined, mean $\pm \mathrm{SD}$ & $17.0 \pm 7.4$ & $19.4 \pm 5.4$ & 0.08 \\
\hline \multicolumn{4}{|l|}{ Biopsy focus score (no. lymphocytic foci $/ 4 \mathrm{~mm}^{2}$ ), } \\
\hline median (quartiles) & $4.3(2.3-5.8)$ & $4.4(2.5-5.8)$ & 0.72 \\
\hline Tarpley biopsy score, median (quartiles) & $3(2-3)$ & $2.5(2-3)$ & 0.98 \\
\hline Fibrosis, median (quartiles) & $1(1-1.8)$ & $1(1-2)$ & 0.64 \\
\hline Fatty cell infiltration, median (quartiles) & $1(1-2.8)$ & $2(1-3)$ & 0.49 \\
\hline Presence of ectopic germinal center (GC), n (\%) & $7(25)$ & $11(39)$ & 0.97 \\
\hline \multicolumn{4}{|l|}{$\begin{array}{l}\text { Prevalence of infiltrating cell types (mean percentage } \\
\text { of total infiltrating MNC or MNC types } \pm \text { SD) }\end{array}$} \\
\hline $\mathrm{CD} 3+\mathrm{T}$ cells & $45.8 \pm 9.8$ & $47.2 \pm 12.3$ & 0.12 \\
\hline CD4+ T cells & $30.8 \pm 7.9$ & $26.9 \pm 10.2$ & 0.02 \\
\hline $\mathrm{CD} 4+/ \mathrm{CD} 3+\mathrm{T}$ cells & $67.1 \pm 9.0$ & $54.6 \pm 13.0$ & 0.02 \\
\hline $\mathrm{CD} 8+\mathrm{T}$ cells & $15.1 \pm 5.2$ & $21.9 \pm 8.3$ & 0.25 \\
\hline CD8+/CD3+ T cells & $32.9 \pm 9.0$ & $45.4 \pm 13.0$ & 0.02 \\
\hline $\mathrm{CD} 4+/ \mathrm{CD} 8+\mathrm{T}$ cell ratio & $2.2 \pm 1.0$ & $1.4 \pm 0.9$ & 0.09 \\
\hline FOXP3+ Treg cells & $1.4 \pm 1.1$ & $2.0 \pm 1.7$ & 0.49 \\
\hline FOXP3+ Treg/CD3+ T cells & $3.0 \pm 2.5$ & $4.4 \pm 3.3$ & 0.44 \\
\hline CD20+ B cells & $46.7 \pm 11.8$ & $44.5 \pm 13.1$ & 0.45 \\
\hline $\mathrm{CD} 3+\mathrm{T} / \mathrm{CD} 20+\mathrm{B}$ cell ratio & $1.1 \pm 0.7$ & $1.3 \pm 1.0$ & 0.16 \\
\hline CD68+ macrophages & $4.5 \pm 3.6$ & $4.7 \pm 3.9$ & 0.33 \\
\hline Fascin+ fDC & $1.5 \pm 1.2$ & $1.9 \pm 1.4$ & 0.57 \\
\hline S100+ iDC & $0.9 \pm 0.8$ & $0.7 \pm 0.5$ & 0.45 \\
\hline CD56+ NK cells & $0.2 \pm 0.5$ & $0.6 \pm 0.6$ & 0.96 \\
\hline
\end{tabular}

MNC: mononuclear cells; fDC: follicular dendritic cell; iDC: interdigitating dendritic cells; NK: natural killer cell. 
Table 3. Progression of grade of MSG autoimmune lesions during followup.

\begin{tabular}{|c|c|c|c|c|c|c|c|}
\hline \multirow{2}{*}{ Patient } & \multirow{2}{*}{$\begin{array}{c}\text { Biopsy } \\
\text { Interval (mo) }\end{array}$} & \multicolumn{3}{|c|}{ Infiltrating $\mathrm{MNC} / \mathrm{mm}^{2}$ of Tissue } & \multicolumn{3}{|c|}{ MSG Biopsy Focus Score } \\
\hline & & 1st Biopsy & 2nd Biopsy & Change, $\%$ & 1st Biopsy & 2nd Biopsy & Change, $\%$ \\
\hline 1 & 77.5 & 668 & 703 & 2.2 & 1.5 & 1.6 & 6.5 \\
\hline 2 & 43.0 & 579 & 530 & -8.5 & 1.0 & 1.00 & 0.00 \\
\hline 3 & 36.0 & 146 & 154 & 5.5 & 1.3 & 1.0 & -23.1 \\
\hline 4 & 42.0 & 748 & 560 & -25.1 & 2.3 & 2.6 & 14.5 \\
\hline 5 & 36.0 & 1001 & 386 & $-61.4^{\dagger}$ & 1.9 & 1.5 & -20.9 \\
\hline 6 & 36.0 & 888 & 831 & -6.4 & 2.3 & 2.3 & -2.2 \\
\hline 7 & 110.0 & 749 & 1105 & 47.5 & 2.7 & 3.3 & 24.7 \\
\hline 8 & 36 & 431 & 1225 & $184.2^{\dagger}$ & 2.2 & 2.5 & 12.4 \\
\hline 9 & 66.0 & 1369 & 1221 & -10.8 & 3.1 & 3.0 & -4.2 \\
\hline 10 & 56.0 & 1827 & 1824 & -0.2 & 4.6 & 5.0 & 9.4 \\
\hline 11 & 52.0 & 908 & 1713 & $88.7^{\dagger}$ & 3.6 & 3.4 & -5.3 \\
\hline 12 & 48.0 & 1198 & 1648 & 37.6 & 3.4 & 3.2 & -4.7 \\
\hline $13^{*}$ & 54.0 & 715 & 1214 & $69.8^{\dagger}$ & 1.8 & 3.1 & $71.1^{\dagger}$ \\
\hline $14^{*}$ & 42.0 & 4781 & 4817 & 0.8 & 5.6 & 6.9 & 24.1 \\
\hline 15 & 109.0 & 2098 & 6383 & $204.2^{\dagger}$ & 4.0 & 11.6 & $190.0^{\dagger}$ \\
\hline 16 & 58.0 & 3575 & 3318 & -7.2 & 5.8 & 4.8 & -17.5 \\
\hline 17 & 89.0 & 2954 & 943 & $-68.1^{\dagger}$ & 6.9 & 2.0 & $-71.1^{\dagger}$ \\
\hline $18 *$ & 41.0 & 3500 & 3522 & 0.6 & 5.9 & 5.8 & -1.4 \\
\hline $19 *$ & 36.0 & 6159 & 5737 & -6.9 & 5.2 & 5.3 & 2.5 \\
\hline $20 *$ & 64.0 & 5809 & 6027 & 3.8 & 6.0 & 6.1 & 2.3 \\
\hline 21 & 82.0 & 5110 & 5856 & 14.6 & 6.3 & 6.4 & 1.6 \\
\hline 22 & 77.0 & 3868 & 3949 & 2.1 & 6.0 & 5.8 & -3.3 \\
\hline 23 & 107.5 & 4522 & 4556 & 0.8 & 5.0 & 5.1 & 2.0 \\
\hline 24 & 82.0 & 2479 & 2667 & 7.6 & 4.0 & 4.0 & 0.0 \\
\hline 25 & 42.0 & 7019 & 9604 & 36.8 & 8.2 & 11.4 & 39.0 \\
\hline 26 & 54.0 & 4654 & 4739 & 1.8 & 5.3 & 5.8 & 8.4 \\
\hline 27 & 89.0 & 4711 & 4462 & -5.3 & 5.7 & 5.5 & -3.0 \\
\hline 28 & 64.0 & 5197 & 5220 & 0.4 & 4.8 & 4.9 & 2.1 \\
\hline
\end{tabular}

$\dagger$ Changes over $60 \%$ or change in Tarpley score. * Patients diagnosed with MSG-associated MALT lymphoma at the second biopsy. MSG biopsy focus score: number of lymphocytic foci per $4 \mathrm{~mm}^{2}$ of tissue.

shown), including the expression of autoantibodies against $\mathrm{Ro} / \mathrm{SSA}$ and/or La/SSB ribonucleoproteins (1 out of 4 patients with GC neo-organization was negative). This can be attributed to the fact that in our sample, GC formation was found not to correlate significantly with the presence of autoantibodies - 9/11 (81.8\%) GC-positive patients versus $13 / 17(76.5 \%)$ GC-negative patients were positive for these autoantibodies $(\mathrm{p}=0.07)$.

Progression of composition of inflammatory MSG lesions of patients with SS. In accord with the poor progression of the infiltration grade, data analysis revealed that the prevalence of the majority of the MSG infiltrating immune cells, including total T cells and Tregs, B cells, macrophages, DC (interdigitating and follicular), and NK cells, was not significantly changed in repetitive biopsies. Only the $\mathrm{CD} 4+/ \mathrm{CD} 3+\mathrm{T}$ cell and $\mathrm{CD} 8+/ \mathrm{CD} 3+\mathrm{T}$ cell percentages were significantly decreased and increased, respectively, during followup. These changes were consistent in all samples, but they were not accompanied by a significant alteration of the $\mathrm{CD} 4+/ \mathrm{CD} 8+\mathrm{T}$ cell ratio (Table 2). The infiltration by the MNC types was not affected by biopsy time interval, histological, demographic, clinical, or therapeutic measures.
Development of MALT lymphoma at the MSG lesions of patients with SS over time. Five patients (Patients 13, 14, 18, 19, and 20) developed MALT lymphoma during followup, as attested by the immunohistochemical and/or molecular detection of B cell monoclonality at the second MSG biopsy. In all 5 patients, lymphoma was restricted to MSG. The development of lymphoma was not associated with expansion of B cells, since their prevalence remained unchanged between the 2 repetitive biopsies (mean percentage of B cells/total infiltrating MNC in the first vs the second biopsy: $40.7 \pm$ SD 15.6 vs $42.3 \pm$ SD 16.6; $p=$ $0.6)$. Further, it was not associated with a fluctuation in the lesion size, since the grade of infiltration remained unaltered in 4 out of 5 patients (Patients 14, 18, 19, and 20; Table 2), nor with severe infiltration or lymphoid organization to GC. MALT lymphoma development was not correlated with biopsy time interval, biopsy scores, number of infiltrating $\mathrm{MNC} / \mathrm{mm}^{2}$ tissue, or the other histological variables tested (data not shown). It correlated with the 2 previously identified clinical adverse prognostic factors ${ }^{9}$, low serum $\mathrm{C} 4$ levels $(\mathrm{p}=0.05)$ and cryoglobulinemia $(p=0.03)$, which were evident at the time of the first biopsy. 


\section{DISCUSSION}

Our findings suggest that the major progression of MSG inflammatory lesions involves the B cell malignant transformation and not a change in the grade and the prevalence of the major infiltrating MNC types. Indeed, even in cases accumulating a 10-year followup, the intensity of the lesions did not change; mild, intermediate, or severe lesions remained the same. This was also observed in specimens that presented a notable fluctuation of the infiltrating MNC number/tissue area or the biopsy focus score during followup. This fluctuation is in agreement with previous data, presenting an alteration of biopsy focus score in $67 \%{ }^{10}$ and $33 \%{ }^{11}$ of patients with primary SS. The differences in the percentage of patients with SS whose biopsy focus score changed over time among the 3 groups can be attributed to the lack of a cutoff value for considering an alteration as significant in the 2 previous studies ${ }^{10,11}$, or to the predominance of patients with severe lesions in our study. Changes in the degree of infiltration were detected only in patients with SS with intermediate and severe lesions, without affecting lesion severity. They were not found to correlate with any histological or clinical factors studied, including the biopsy time interval and the therapeutic interventions applied. This is in accord with previous data showing that treatment with corticosteroids or disease-modifying antirheumatic drugs (DMARD) does not modify the salivary flow rate of patients with $\mathrm{SS}^{12}$. The change of infiltrating CD4+ and CD8+ T cell subpopulations was rather mild, since it did not affect the CD4+/CD8+ T cell ratio. Histological indicators of tissue degeneration, such as fibrosis and fatty cell infiltration, remained unchanged over time, a fact also observed in heavily infiltrated tissues, suggesting that the chronic inflammation in SS does not necessarily lead to degeneration of the glandular tissue and replacement by fibrosis and fatty cells, as assumed ${ }^{13}$. These findings, along with previous data showing insignificant histological deterioration of the liver lesions of SS patients with primary biliary cirrhosis ${ }^{14}$, indicate that the SS autoimmune lesions are slowly progressing. Further, the histological findings are consistent with previous assessments of salivary function showing that the unstimulated saliva flow rates do not change over time ${ }^{10,12}$; only the stimulated ones worsen ${ }^{12}$ in patients with SS.

In 24 out of the 28 patients with SS, the first MSG biopsy was done at diagnosis and remained generally unaltered thereafter, suggesting that fully developed MSG lesions occur at diagnosis. The abiding histopathologic features of the MSG inflammatory lesions over time imply the operation of homeostatic mechanisms that contribute to survival of the inflamed epithelia and tightly regulate the size and composition of the invariable inflammatory infiltrates ${ }^{13}$. Epithelial cells, which are major regulators of local immune responses ${ }^{15}$, may determine the extent of inflammation. The fully developed MSG lesions at diagnosis and their minor modification thereafter suggest that the lesion course follows the clinical picture of the disease. In SS, several clinical and laboratory indices, including adverse prognostic factors for lymphoma development, such as salivary gland (SG) enlargement, palpable purpura, C3 or C4 hypocomplementemia, and cryoglobulinemia, are evident at diagnosis and do not change significantly thereafter ${ }^{1,9,13}$.

One of the noteworthy findings in our study was the development of MALT lymphoma in 5 patients during followup. The elevated percentage of patients that developed B cell transformation possibly relates to the high number of patients with severe inflammation that were included in our study. However, this transformation was found not to associate with any histological factors, including GC formation ${ }^{13}$, but only to previously identified laboratory measures such as low serum $\mathrm{C} 4$ levels and cryoglobulinemia. Although our study did not reveal any correlation with histological variables and the offending factors for this transformation remain unknown, our findings suggest that in high-risk patients, an MSG biopsy must be repeated in order to diagnose development of lymphoma.

The lack of progression of histological features of MSG biopsy over time does not signify simply its diagnostic value, but suggests that it might be useful for patient classification. The fixed $\mathrm{T}$ or $\mathrm{B}$ cell predominance suggests that patients with SS can be classified into distinct subgroups according to the dominating immune responses. This is of major significance for the selection of appropriate targeted therapeutic approaches, e.g., B cell depletion therapy in patients with SS with predominating B cell responses at the inflammatory lesions.

Our findings indicate that in the majority of patients with SS, progression of MSG autoimmune lesions is rather slow. However, in a subgroup of SS patients expressing adverse serological prognostic factors, the MSG infiltrates progress and MALT lymphoma develops. This progression is not accompanied by changes in the grade and incidence of the major infiltrating MNC types. These findings suggest that, except in cases of suspected development of lymphoma, repeated evaluation of MSG biopsies is unnecessary and underline the value of MSG biopsy for the selection of an appropriate therapy.

\section{REFERENCES}

1. Tzioufas AG, Kapsogeorgou EK, Moutsopoulos HM. Pathogenesis of Sjogren's syndrome: What we know and what we should learn. J Autoimmun 2012;39:4-8.

2. Christodoulou MI, Kapsogeorgou EK, Moutsopoulos HM. Characteristics of the minor salivary gland infiltrates in Sjogren's syndrome. J Autoimmun 2010;34:400-7.

3. Vitali C, Bombardieri S, Jonsson R, Moutsopoulos HM, Alexander EL, Carsons SE, et al. Classification criteria for Sjogren's syndrome: A revised version of the European criteria proposed by the American-European Consensus Group. Ann Rheum Dis 
2002;61:554-8

4. Al-Hashimi I, Wright JM, Cooley CA, Nunn ME. Reproducibility of biopsy grade in Sjogren's syndrome. J Oral Pathol Med 2001;30:408-12.

5. Morbini P, Manzo A, Caporali R, Epis O, Villa C, Tinelli C, et al. Multilevel examination of minor salivary gland biopsy for Sjogren's syndrome significantly improves diagnostic performance of AECG classification criteria. Arthritis Res Ther 2005;7:R343-8.

6. Chisholm DM, Mason DK. Labial salivary gland biopsy in Sjogren's disease. J Clin Pathol 1968;21:656-60.

7. Tarpley TM Jr, Anderson LG, White CL. Minor salivary gland involvement in Sjogren's syndrome. Oral Surg Oral Med Oral Pathol 1974;37:64-74.

8. Skopouli FN, Li L, Boumba D, Stefanaki S, Hanel K, Moutsopoulos HM, et al. Association of mast cells with fibrosis and fatty infiltration in the minor salivary glands of patients with Sjogren's syndrome. Clin Exp Rheumatol 1998;16:63-5.

9. Voulgarelis M, Tzioufas AG. Pathogenetic mechanisms in the initiation and perpetuation of Sjogren's syndrome. Nat Rev Rheumatol 2010;6:529-37.
10. Jonsson R, Kroneld U, Backman K, Magnusson B, Tarkowski A. Progression of sialadenitis in Sjogren's syndrome. Br J Rheumatol 1993;32:578-81.

11. Leroy JP, Pennec YL, Soulier C, Berthelot JM, Letoux G, Youinou P. Follow up study of labial salivary gland lesions in primary Sjogren's syndrome. Ann Rheum Dis 1992;51:777-80.

12. Pijpe J, Kalk WW, Bootsma H, Spijkervet FK, Kallenberg CG, Vissink A. Progression of salivary gland dysfunction in patients with Sjogren's syndrome. Ann Rheum Dis 2007;66:107-12.

13. Jonsson R, Vogelsang P, Volchenkov R, Espinosa A, Wahren-Herlenius M, Appel S. The complexity of Sjogren's syndrome: Novel aspects on pathogenesis. Immunol Lett 2011;141:1-9.

14. Hatzis GS, Fragoulis GE, Karatzaferis A, Delladetsima I, Barbatis C, Moutsopoulos HM. Prevalence and longterm course of primary biliary cirrhosis in primary Sjogren's syndrome. J Rheumatol 2008;35:2012-6.

15. Manoussakis MN, Kapsogeorgou EK. The role of intrinsic epithelial activation in the pathogenesis of Sjogren's syndrome. J Autoimmun 2010;35:219-24. 